\title{
The Impact of Pre-transplant Cell-free DNA Levels on Leukemia Relapse and Transplant-related Complications in Allogeneic Hematopoietic Stem Cell Transplant Recipients
}

\author{
Zeynep Arzu Yegin1, Ferda Can1, Sanem Gökçen1, Rezzan Eren Sadioğlu², Zübeyde Nur Özkurt1, \\ Çiğdem İlhan1, Münci Yağc1 1
}

\author{
${ }^{1}$ Department of Hematology, Gazi University School of Medicine, Ankara, Turkey \\ ${ }^{2}$ Department of Internal Medicine, Gazi University School of Medicine, Ankara, Turkey
}

Background: Cell-free DNA, which may be considered as "liquid" biopsy, may serve as a diagnostic and prognostic marker not only in hematological malignancies but in solid tumors as well.

Aims: To investigate the prognostic role of pre-transplant cell-free DNA levels in allogeneic hematopoietic stem cell transplant recipients. Study Design: Retrospective cohort study.

Methods: A total of 177 allogeneic hematopoietic stem cell transplant recipients [median age: 36 (16-66) years; male/female: 111/66] with an initial diagnosis of acute leukemia were included in the study. Cell-free DNA was extracted from pre-transplant serum samples by using the MagNA Pure Compact Nucleic Acid Isolation Kit I with the MagNA Pure Compact instrument (Roche Diagnostics, Penzberg, Germany).

Results: A positive correlation was demonstrated between cell-free DNA and age $(\mathrm{p}=0.018 ; \mathrm{r}=0.177)$. Pre-transplant cell-free DNA levels were lower in bcr-abl $(+)$ patients $(\mathrm{p}=0.001)$, while an adverse correlation was indicated between cell-free DNA and bcr-abl levels $(\mathrm{p}=0.001 ; \mathrm{r}=-0.531)$. Acute lymphoblastic leukemia patients with bcr-abl positivity $(\mathrm{p}=0.001)$ or abnormal cytogenetics $(\mathrm{p}=0.038)$ represented significantly lower pre-transplant cell-free DNA levels. Cell-free DNA levels were lower in patients who developed sinusoidal obstruction syndrome $(\mathrm{p}=0.035)$. In terms of long-term complications, acute myeloid leukemia patients who experienced post-transplant relapse had significantly lower pre-transplant cell-free DNA levels $(\mathrm{p}=0.024)$. Overall survival was not statistically different between high- and low- cell-free DNA groups ( $45.2 \%$ vs 22.5 ; $\mathrm{p}=0.821$ ).

Conclusion: In general, low serum levels of pre-transplant çellfree DNA seem to be associated with transplant-related morbidities and may be considered an adverse prognostic factor for allogeneic hematopoietic stem cell transplant recipients.

Keywords: Acute lymphoblastic leukemia, acute myeloid leukemia, allogeneic hematopoietic stem cell transplantation, cell-free DNA
Hematological malignancies may be considered as "liquid" tumors, because the primary involved sites are bone marrow and lymphoid tissues, along with neoplastic cells circulating in the peripheral blood (1). The amount of cell-free deoxyribonucleic acid (cfDNA) increased in patients with hematological malignancies besides a variety of solid tumors. Circulating cfDNA may sustain genetic characteristics of the tumor as cancer-specific mutations were demonstrated in the circulating cfDNA of acute myeloid leukemia (AML) and myelodysplastic syndrome (MDS) patients. In addition, tumor-associated micro-ribonucleic acids were identified in the peripheral blood of lymphoma patients $(2,3)$. Circulating cfDNA, which may be described as "liquid" biopsy, is currently introduced as a diagnostic marker because it provides a typical representation of tumor genetic profile and has a feasible role in minimal residual disease (MRD) determination, which makes it a potential predictor of prognosis $(2,4-7)$.

Cell-free DNA is composed of extracellular DNA fragments that are released from the cells as a result of necrosis or apoptosis. In healthy individuals, cfDNA is a product of physiological hematopoietic cell cycle, including myeloid and lymphoid apoptotic cells, whereas in cancer patients, only a small amount of total cfDNA originates from neoplastic cells. The wide range of this circulating tumor DNA fraction, which differs between $0.01 \%$ and $60 \%$ of total cfDNA, depends on several tumoral features, including volume, stage, vascularization, proliferation, and apoptosis rate (1,2,4,7-9).

To date, the predictor role of cfDNA was demonstrated in a wide spectrum of malignancies, including hematological disorders such as AML, MDS, and lymphoid neoplasms (2). In particular, this

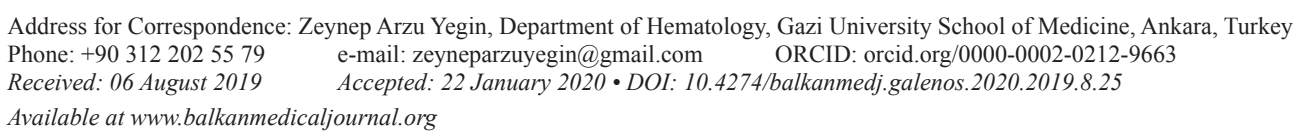


study was performed to evaluate the potential prognostic role of pre-transplant cfDNA levels in the development of transplantrelated complications and survival in allogeneic hematopoietic stem cell transplantation (allo-HSCT) recipients.

\section{MATERIALS AND METHODS}

The study cohort was composed of 177 acute leukemia patients who underwent allo-HSCT. Participants were classified into two subgroups for comparable statistical analysis, which were denominated as high- and low-cfDNA groups, based on the median cfDNA level, which was considered to be 8.5 (3.7-56.6) ng/ $\mu \mathrm{L}$. Patient demographics and transplant characteristics are presented in Tables 1 and 2.

Pre-transplant serum samples were stored at $-80^{\circ} \mathrm{C}$ until the assay. Cell-free DNA was obtained from the serum on the basis of the manufacturer's instructions by using MagNA Pure Compact Nucleic Acid Isolation Kit I and instrument (Roche Diagnostics, Penzberg, Germany). Four hundred microliters of serum specimen were used as the starting volume, and the nucleic acids were eluted in $100 \mu \mathrm{L}$ of the supplied elution buffer. Cell-free DNA concentration, which is expressed in $n g / \mu \mathrm{L}$, was measured at 260

TABLE 1. Patient and disease characteristics

\begin{tabular}{|c|c|}
\hline Age [median (range])] (years) & $36(16-66)$ \\
\hline Gender (n \%) (male/female) & $111 / 66$ \\
\hline \multicolumn{2}{|l|}{ Diagnosis (n \%) } \\
\hline Acute myeloid leukemia & $99(55.9)$ \\
\hline Acute lymphoblastic leukemia & $74(41.8)$ \\
\hline Mixed-phenotype acute leukemia & $3(1.7)$ \\
\hline Blastic plasmacytoid dendritic cell neoplasm & $1(0.6)$ \\
\hline Therapy-related acute leukemia & - \\
\hline Extramedullary disease (n \%) & $17(9.6)$ \\
\hline CNS involvement (n \%) & $5(2.8)$ \\
\hline \multicolumn{2}{|l|}{ Cytogenetics (n \%) $(n=76)$} \\
\hline Normal & $32(42.1)$ \\
\hline Abnormal & $44(57.9)$ \\
\hline Complex karyotype & $10(22.7)$ \\
\hline Other & $34(77.3)$ \\
\hline \multicolumn{2}{|l|}{ FLT3 Mutational Status for AML (n \%) $(n=19)$} \\
\hline Positive & - \\
\hline Negative & $19(100)$ \\
\hline \multicolumn{2}{|l|}{ NPM-1 Mutational Status for AML (n \%) $(n=20)$} \\
\hline Positive & $13(65)$ \\
\hline Negative & $7(35)$ \\
\hline \multicolumn{2}{|c|}{ AML1-ETO (RUNX1/RUX1T1) for AML [n (\%)] $(n=26)$} \\
\hline Positive & $11(42.3)$ \\
\hline Negative & $15(57.7)$ \\
\hline \multicolumn{2}{|l|}{ Inv16 (CBFB/MYH11) for AML $(n[\%])(n=21)$} \\
\hline Positive & $1(4.8)$ \\
\hline Negative & $20(95.2)$ \\
\hline \multicolumn{2}{|l|}{ Bcr-abl (n \%) (n=39) } \\
\hline Positive & $17(43.6)$ \\
\hline Negative & $22(56.4)$ \\
\hline
\end{tabular}

AML: acute myeloid leukemia, CNS: central nervous system, FLT3: fms like tyrosine kinase 3, NPM-1: nucleophosmin $\mathrm{nm}$ and the purity was measured using the $260 / 280$ ratio by using a Thermo Scientific ${ }^{\mathrm{TM}}$ NanoDrop Lite Spectrophotometer.

Median pre-transplant cfDNA level was 8.5 (3.7-56.6) $\mathrm{ng} / \mu \mathrm{L}$, whereas simultaneous measurement of white blood cell (WBC) count yielded a median of $4.6(0.3-17.7) \times 109 / \mathrm{L}$ in the whole population. Given that WBC counts may interfere with cfDNA analysis, we performed a comparative analysis and did not find any significant statistical correlation between cfDNA levels and WBC counts. WBC counts were similar in high- and low-cfDNA groups. Therefore, additional processing such as logarithmic transformation was not required for statistical analysis.

\section{Statistical analysis}

All 177 subjects had valid cfDNA data with a mean serum level of $9.576 \mathrm{ng} / \mu \mathrm{L}$. On the basis of a standard deviation of 5.857, the standard error was estimated to be 0.440 . The lower and upper boundaries of the $95 \%$ confidence interval (CI) were between 8.707 and $10.444(p<0.001)$. The $95 \%$ CIs for cumulative survival were determined to be between -8.325 and $10.483(p=0.821)$. The statistical power of the study was $95.23 \%$ on post hoc analysis. Shapiro-Wilk test was performed for normality analysis. Parametric and non-parametric tests were used in case of normal

TABLE 2. Transplant characteristics

\begin{tabular}{ll}
\hline Time to transplant [median (range)] (months) & $5.6(1-125.1)$ \\
Pre-transplant disease status (n [\%]) & $154(87)$ \\
$\quad$ Complete remission & $6(3.4)$ \\
$\quad$ Partial remission & $17(9.6)$ \\
$\quad$ Refractory & \\
Donor type (n [\%]) & $144(81.4)$ \\
$\quad$ Related & $30(16.9)$ \\
$\quad$ Unrelated & $3(1.7)$ \\
$\quad$ Haploidentical & \\
HLA match status (n [\%]) & $149(84.2)$ \\
$\quad$ Full-matched & $28(15.8)$ \\
$\quad$ Mismatched & \\
Graft type (n [\%]) & - \\
$\quad$ Bone marrow & $177(100)$ \\
$\quad$ Peripheral blood & \\
Conditioning regimen (n [\%]) & $160(90.4)$ \\
$\quad$ Myeloablative & $17(9.6)$ \\
$\quad$ Reduced intensity & $68(38.4)$ \\
Total body irradiation (n [\%]) & \\
GvHD Prophylaxis (n [\%]) & $161(91)$ \\
$\quad$ Cyclosporine A + methotrexate & $13(7.3)$ \\
Cyclosporine A + mycophenolate mofetil & $3(1.7)$ \\
Cyclosporine A + methotrexate + ATG & $36 / 177$ \\
Defibrotide prophylaxis (n [\%]) & $3(0-18)$ \\
Number of febrile days (median [range]) & $4.4(0.8-7.3)$ \\
Infused CD34+ cells (median [range]) (106/kg) & $16(0-30)$ \\
Neutrophil engraftment (median [range]) (days) & $14(0-67)$ \\
Platelet engraftment (median [range]) (days) & \\
\hline ATG: antithymocyte globulin, GvHD: graft versus host disease & \\
\hline & \\
&
\end{tabular}


and abnormal distribution, respectively. For normal distributed groups, equality and homogeneity of variances were analyzed by Levene's test through one-way ANOVA and Student's t-tests. Continuous variables were compared by conducting Student's t-test for normal distribution, whereas Mann-Whitney U and Kruskal-Wallis tests were used for abnormal distributed parameters. Categorical variables were analyzed by chi-square test. Correlations were determined by Pearson and Spearman tests for normal and abnormal distributed data, respectively. Survival analysis was performed using Kaplan-Meier test. Cox regression and logrank tests were used for the assessment of risk factors that were significantly associated with survival. The statistical threshold for significance was considered to be $\mathrm{p}<0.05$ on SPSS 22.0 (SPSS Inc, Chicago, IL, USA).

\section{Ethical standards}

The study was certified by the institutional ethics board of the Gazi University Faculty of Medicine (date: 12.02.2018; number: 106). The procedures in the study were consistent with the Helsinki Declaration, and informed consent was obtained from all participants.

\section{RESULTS}

Median cfDNA levels did not statistically differ in terms of gender; primary diagnosis; extramedullary leukemia; cytogenetics, including fms like tyrosine kinase 3 (FLT3), nucleophosmin (NPM-1), AML1-ETO (RUNX1/RUX1T1), inv16 (CBFB/MYH11), and complex karyotype; pre-transplant disease status, development of mucositis; peri-engraftment infections; cytomegalovirus (CMV) reactivation; thrombotic microangiopathy (TMA); graft versus host disease (GvHD); and relapse $(\mathrm{p}>0.05)$. Early and late transplant complications are detailed in Table 3.

Patients who presented bcr-abl positivity had significantly lower cfDNA levels than bcr-abl (-) patients [7.5 (5.4-12.1) vs 9.5 (7-13.4) $\mathrm{ng} / \mu \mathrm{L} ; \mathrm{p}=0.001]$. A negative correlation was identified between bcr-abl status and cfDNA amount $(\mathrm{p}=0.001 ; \mathrm{r}=-0.531)$. Patients with sinusoidal obstruction syndrome (SOS) had lower levels of cfDNA compared with patients who did not develop SOS [7.7 (4.7-15.3) vs $8.8(5.2-56.6) \mathrm{ng} / \mu \mathrm{L} ; \mathrm{p}=0.035]$. In addition, a tendency to SOS was observed in patients who had lower pretransplant cfDNA levels $(\mathrm{p}=0.049)$. An adverse relationship existed between cfDNA amount and SOS development $(p=0.035$; $\mathrm{r}=-0.163$ ). Cytomegalovirus reactivation was also more frequent in patients with lower pre-transplant cfDNA levels, without statistical significance $(\mathrm{p}=0.065)$.

Peri-transplant infections were evaluated in 153 patients. Febrile neutropenia was demonstrated in 134 patients (87.6\%). Thirtyseven patients $(27.6 \%)$ had catheter-related infections, 19 patients $(14.2 \%)$ had bloodstream infections, 15 patients $(11.2 \%)$ had urinary tract infections, 11 patients $(8.2 \%)$ had pneumonia, and 21 patients $(15.7 \%)$ had other types of infections. In a total of 31 patients $(23.1 \%)$, the site of infection or microbiological documentation was not identified. Seven patients developed invasive fungal infection. Any significant association was not indicated between cfDNA levels and infectious complications.
Median age was higher in the high-cfDNA group than in the lowcfDNA group [41 (18-65) years vs 29 (16-66) years; $p=0.03$ ]. A positive correlation existed between age and pre-transplant cfDNA levels $(\mathrm{p}=0.018 ; \mathrm{r}=0.177)$. Neutrophil and platelet engraftment days, chimerism values, frequency of SOS, mucositis, TMA, infectious morbidity, hemorrhagic cystitis, acute/chronic GvHD, and post-transplant relapse were not significantly different among high- and low-cfDNA groups ( $p>0.05$ ). The total number of febrile days was lower in patients with high cfDNA levels than in the lowcfDNA group [2 (0-15) days vs $3(0-18)$ days; $p=0.073$ ].

In the subgroup analysis of AML patients, significantly lower pre-transplant cfDNA levels were observed in the course of post-transplant relapse when compared with those who stayed in remission [8 (5.2-11.5) vs $9.6(3.7-14.5) \mathrm{ng} / \mu \mathrm{L} ; \mathrm{p}=0.024)$ (Figure 1). No significant association was observed between cfDNA levels and cytogenetical features, including complex karyotype and the mutational status of FLT3, NPM-1, AML1-ETO (RUNX1/ RUX1T1), and inv16 (CBFB/MYH11).

In the acute lymphoblastic leukemia (ALL) group, bcr-abl (+) patients had significantly lower pre-transplant cfDNA levels than bcr-abl (-) patients [7.5 (5.4-12.1) vs $9.7(7-13.4) \mathrm{ng} / \mu \mathrm{L} ; \mathrm{p}=0.001]$ (Figure 2). Similarly, cfDNA levels were lower in patients who presented abnormal cytogenetics compared with patients with

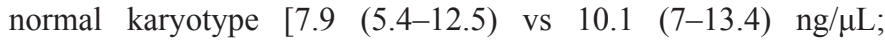
$\mathrm{p}=0.038]$. SOS was observed more frequently in the low-cfDNA group $(\mathrm{p}=0.019)$.

Overall survival (OS) was $15.3 \%$ in the whole population at the the end of $14.6(0.2-160.9)$ months of follow-up. OS was not

TABLE 3. Early and late complications

\begin{tabular}{|c|c|}
\hline Sinusoidal obstruction syndrome (n \%) & $41(23)$ \\
\hline Grade $[$ median (range)] & $1(0-4)$ \\
\hline Mucositis (n \%) & $163(92.1)$ \\
\hline Grade [median (range)] & $2(1-4)$ \\
\hline Pre-engraftment infections ( $\mathrm{n} \%$ ) & $134(75.7)$ \\
\hline $\begin{array}{l}\text { Thrombotic microangiopathy ( } \mathrm{n} \% \text { ) (in the first } 100 \\
\text { days) }\end{array}$ & $11(6.2)$ \\
\hline CMV reactivation (n \%) (in the first 100 days) & $51(28.8)$ \\
\hline Viral hemorrhagic cystitis ( $\mathrm{n} \%$ ) (in the first 100 days) & $25(14.1)$ \\
\hline Acute GvHD (n \%) & $71(40.1)$ \\
\hline Grade [median (range)] & $2(1-4)$ \\
\hline Onset [median (range)] (days) & $50(7-372)$ \\
\hline \multicolumn{2}{|l|}{ Sites $(\mathrm{n} \%)$} \\
\hline Skin & $36(50.7)$ \\
\hline Liver & $3(4.2)$ \\
\hline Gastrointestinal tract & $14(19.7)$ \\
\hline More than one site & $18(25.4)$ \\
\hline Chronic GvHD (n \%) & $67(37.8)$ \\
\hline Post-transplant lymphoproliferative disorder (n \%) & $1(0.6)$ \\
\hline Secondary malignancy (n \%) & $2(1.1)$ \\
\hline Graft failure (n \%) & $2(1.1)$ \\
\hline Relapse (n \%) & $63(35.6)$ \\
\hline Post-transplant day of relapse [median (range)] & $117(30-1460)$ \\
\hline
\end{tabular}


statistically different between high- and low-cfDNA groups (45.2\% vs $22.5 \%$; $\mathrm{p}=0.821$ ) (Figure 3 ).

Univariate Cox regression analysis showed that primary diagnosis $(\mathrm{p}=0.042)$, pre-transplant disease status $(\mathrm{p}=0.004), \operatorname{SOS}(\mathrm{p}<0.001)$, acute GvHD $(p=0.001)$, chronic GvHD $(p<0.001)$, and relapse $(p<0.001)$ have a significant impact on OS. The significance was confirmed for primary diagnosis $(p=0.042)$, SOS $(p<0.001)$, acute GvHD ( $p=0.001)$, chronic GvHD $(p=0.009)$, and post-transplant relapse $(p=0.003)$ in multivariate Cox regression analysis. Pretransplant cfDNA levels did not present any significant impact on OS $(\mathrm{p}>0.05)$.

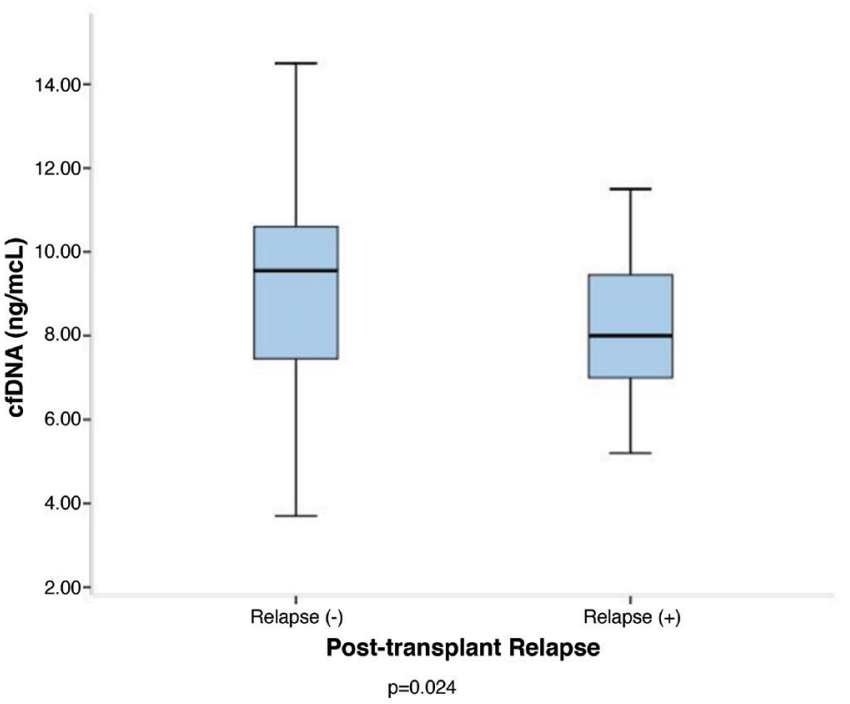

FIG. 1. Pre-transplant cfDNA levels were significantly lower in AML patients who experienced post-transplant relapse compared with the patients who stayed in remission $(\mathrm{p}=0.024)$.

AML: acute myeloid leukemia, cfDNA: cell-free deoxyribonucleic acid

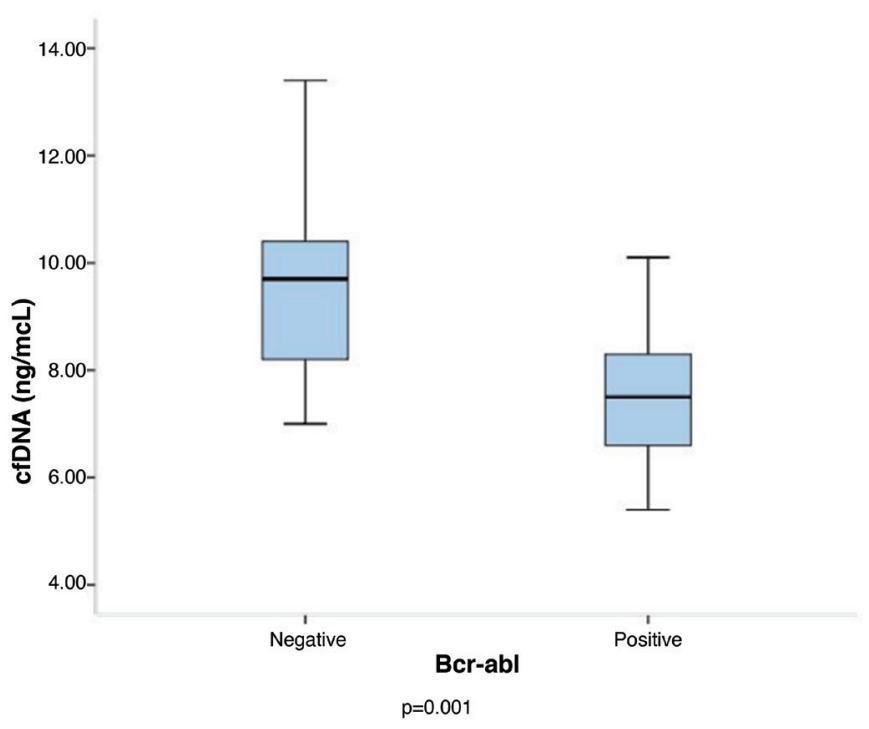

FIG. 2. Pre-transplant cfDNA levels were significantly lower in bcr-abl(+) ALL patients compared with bcr-abl(-) patients $(p=0.001)$.

ALL: acute lymphoblastic leukemia

\section{DISCUSSION}

In this study, the prognostic role of pre-transplant cfDNA levels was investigated in allo-HSCT recipients with acute leukemia. A significant association was identified between cfDNA and age. Pre-transplant cfDNA levels were lower in bcr-abl $(+)$ patients, indicating an adverse correlation between cfDNA and bcr-abl levels. ALL patients with bcr-abl positivity or abnormal cytogenetics presented significantly lower pre-transplant cfDNA levels. Cell-free DNA levels were lower in patients with SOS. In terms of long-term complications, AML patients who presented post-transplant relapse had significantly lower pre-transplant cfDNA levels. OS was not statistically different between high- and low-cfDNA groups. In general, lower serum levels of pre-transplant cfDNA seem to be associated with transplant-related morbidities and may be considered an adverse predictor of prognosis in alloHSCT recipients.

The amount of cfDNA ranges between 10 to $100 \mathrm{ng} / \mathrm{mL}$ in healthy individuals and may be influenced by age, gender, food intake, and diurnal variations. It can circulate as a single molecule or in a complex with cellular or non-cellular components to act as a signaling molecule. Aside from several physiological states such as pregnancy and physical activity, pathological conditions, including inflammation and autoimmune diseases, may present with elevated cfDNA levels. In cancer, cfDNA levels may be increased up to 1000 $\mathrm{ng} / \mathrm{mL}$, which draws attention to an association between cfDNA levels and tumor burden (1,4,8,10-13). A previously reported association between age and cfDNA was confirmed in our study. Teo et al. showed a remarkable consistency between nucleosome signals of cfDNA and redistribution of heterochromatin, which has a role in cellular senescence and aging. In this perspective, cfDNA may be designated as a biomarker of age or a predictor of health status (14).

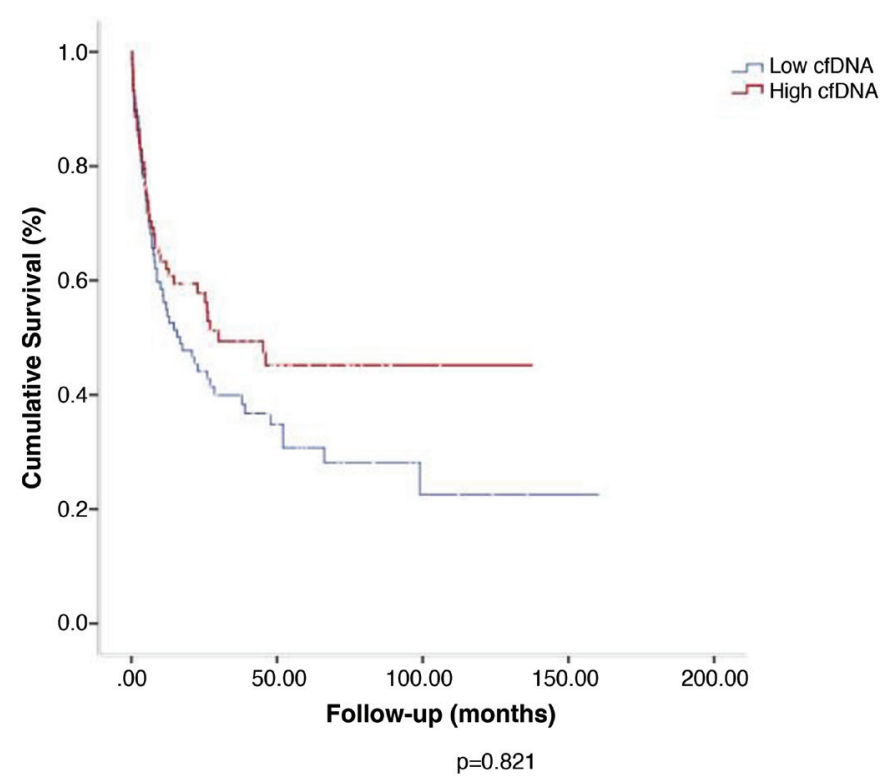

FIG. 3. Overall survival was not statistically different between high- and low-cfDNA groups ( $45.2 \%$ vs $22.5 \%$; $\mathrm{p}=0.821)$.

cfDNA: cell-free deoxyribonucleic acid 
Circulating tumor DNA analysis requires distinctive attention due to its molecular properties, including low concentration and high fragmentation rate, as well as contamination potential with genomic DNA. The methodologies of cfDNA-based analysis need to be improved because the appropriate source of cfDNA and technical details of sample processing have not been clarified (9). Serum levels of cfDNA are 2-24 times more than plasma levels as a result of the contaminaton of genomic DNA, which is considered a product of WBC lysis during serum processing. As serum cfDNA levels are expected to be more variable than plasma levels, higher serum cfDNA levels are suggested to possibly represent an indirect tumor-related process, whereas plasma levels may be a better indicator of in vivo circulating DNA $(4-6,9,15,16)$. Alongside WBC count, several factors may affect cfDNA analysis, such as individualized variations, diurnal rhythm, inflammatory responses, and physical stimuli (1). Although sample preference would be considered an eventual limitation for the present study, we did not mean to discriminate the impact of WBCs on the entire process, considering the potential role of leukocytes in the leukemia microenvironment. From a global perspective, cfDNA may not only be a tumoral or genetic predictor, but also an indicator of inflammation and cytokine stimulation, which is well known to be closely associated with leukocyte activation. The diversity in the methodologies of cfDNA analysis, compared with polymerase chain reaction or next-generation sequencing, may be considered a possible explanation for the contradictory results with the previous studies $(5,17,18)$.

Serum cfDNA levels were incompatible with plasma levels in the previous reports. In a study by Thijssen et al. (15) on patients with colorectal cancer, serum cfDNA was significantly associated with liver metastasis, whereas plasma cfDNA was predictive for recurrence. In concordance with our results, cfDNA levels were decreased in the sera of rheumatoid arthritis (RA) patients. By contrast, several studies stated elevated serum or plasma cfDNA levels in RA patients. Aside from the physiopathological diversity of underlying autoimmune disorders and distinct stages of disease activity at the time of sampling, the role of the heterogeneous methods that were used in sample collection/processing, cfDNA extraction, and quantification should be emphasized. This point of view, which is mainly based on the lack of uniformity and standardization of cfDNA analysis, may lead to a distinct perspective that may particularly elucidate the predictor impact of lower cfDNA levels in the present study (11-13).

Several studies underlined the predictor role of cfDNA in endotheliopathies, which shows a significant association of cfDNA with endothelial markers, including syndecan-1 and thrombomodulin $(19,20)$. Endothelial dysfunction, which is indicated to be the major underlying physiopathological mechanism of SOS, may be a reasonable explanation for the association of SOS and pre-transplant cfDNA levels in the current study. However, the predictive role of lower cfDNA levels and its negative correlation with SOS require further evaluation. Sample preference and insufficient number of patients with SOS may partially help elucidate the contradictory results. Simultaneous measures of cfDNA may be more informative for the clarification of the predictive role of cfDNA in the course of SOS.
Several infectious and inflammatory conditions may present with elevated cfDNA levels, which were shown to be correlated with disease severity and mortality $(21,22)$. In addition, cfDNA was associated with the degree of inflammation in viral infections (23). In consideration with these preliminary data, the insignificant association of cfDNA with CMV reactivation may require further verification.

Cell-free DNA levels were elevated in various hematological disorders. High levels of cfDNA and DNA integrity index represented a lower probability of progression-free survival in diffuse large B cell lymphoma patients (8). Cell-free DNA levels were increased in lymphoma patients and correlated with advanced age and stage, poor prognosis, B symptoms, and lactate dehydrogenase levels $(4,6,24)$. The amount of cfDNA was also higher in myeloma patients; this higher amount was shown to be associated with progressive disease $(4,25,26)$.

Several studies reported high cfDNA levels in acute leukemia patients. Cytogenetic and molecular abnormalities such as FLT3 and NPM-1 were identified in cfDNA, suggesting a feasible role for cfDNA in predicting AML prognosis and treatment indications (4). Gao et al. (27) found that not only cfDNA concentrations but also DNA integrity index were elevated in patients with acute leukemia compared with healthy individuals. Given that the DNA integrity index was correlated with disease status, its clinical utility for MRD assessment may also be considered (27). We did not observe any significant association of cfDNA with AML mutational status. However, the adverse correlation of cfDNA with bcr-abl levels and abnormal cytogenetics in ALL patients should be confirmed with large and prospective studies.

Chimerism analysis may be performed in cfDNA in allo-HSCT recipients. Furthermore, because chimerism analysis of cfDNA was shown to predict early relapse in patients with hematological malignancies, monitoring allo-HSCT survivors with cfDNA could be feasible in predicting early relapse $(28,29)$. Lymphoma patients were shown to have detectable cfDNA prior to progression after allo-HSCT (30). The impact of lower cfDNA levels on posttransplant relapse in AML patients and its statistically insignificant impact on OS, which may be associated with inadequate number of patients, require further verification.

In conclusion, cfDNA, which can be considered a non-invasive and repeatable analysis of genetic profiles, may serve a potential tool for diagnosis and prognosis assessment $(2,7)$. Nevertheless, several issues remain to be solved, including standardization of sample collection, processing, and lack of comparability due to inconsistent thresholds (7). We need further understanding of the biological features of cfDNA to determine its true physiological role.

Conflict of Interest: No conflict of interest was declared by the authors.

Financial Disclosure: No financial disclosure was declared by the authors.

\section{REFERENCES}

1. Buedts L, Vandenberghe P. Circulating cell-free DNA in hematological malignancies. Haematologica 2016;101:997-9.

2. Hocking J, Mithraprabhu S, Kalff A, Spencer A. Liquid biopsies for liquid tumors: emerging potential of circulating free nucleic acid evaluation for the management of hematologic malignancies. Cancer Biol Med 2016;13:215-25. 
3. Fettke H, Kwan EM, Azad AA. Cell-free DNA in cancer: current insights. Cell Oncol (Dordr) 2019;42:13-28.

4. Kubaczkova V, Vrabel D, Sedlarikova L, Besse L, Sevcikova S. Cell-free DNAMinimally invasive marker of hematological malignancies. Eur J Haematol 2017;99:291-9.

5. Kristensen LS, Hansen JW, Kristensen SS, Tholstrup D, Harsløf LB, Pedersen OB, et al. Aberrant methylation of cell-free circulating DNA in plasma predicts poor outcome in diffuse large B cell lymphoma. Clin Epigenetics 2016;8:95.

6. Kurtz DM, Scherer F, Jin MC, Soo J, Craig AFM, Esfahani MS, et al. Circulating Tumor DNA Measurements As Early Outcome Predictors in Diffuse Large B-Cell Lymphoma. J Clin Oncol 2018;36:2845-53.

7. Wu FT, Lu L, Xu W, Li JY. Circulating tumor DNA: clinical roles in diffuse large B cell lymphoma. Ann Hematol 2019;98:255-69.

8. Li M, Jia Y, Xu J, Cheng X, Xu C. Assessment of the circulating cell-free DNA marker association with diagnosis and prognostic prediction in patients with lymphoma: a single-center experience. Ann Hematol 2017;96:1343-51.

9. Martignano F. Cell-free DNA: An overview of sample types and isolation procedures. Methods Mol Biol 2019;1909:13-27.

10. Barták BK, Kalmár A, Galamb O, Wichmann B, Nagy ZB, Tulassay Z, et al. Blood collection and cell-Free DNA isolation methods influence the sensitivity of liquid biopsy analysis for colorectal cancer detection. Pathol Oncol Res 2019;25:915-23.

11. Dunaeva M, Buddingh' BC, Toes RE, Luime JJ, Lubberts E, Pruijn GJ. Decreased serum cell-free DNA levels in rheumatoid arthritis. Auto Immun Highlights 2015;6:23-30.

12. Meddeb R, Dache ZAA, Thezenas S, Otandault A, Tanos R, Pastor B, et al. Quantifying circulating cell-free DNA in humans. Sci Rep 2019;9:5220.

13. Duvvuri B, Lood C. Cell-Free DNA as a biomarker in autoimmune rheumatic diseases. Front Immunol 2019;10:502.

14. Teo YV, Capri M, Morsiani C, Pizza G, Faria AMC, Franceschi C, et al. Cell-free DNA as a biomarker of aging. Aging Cell 2019;18:e12890.

15. Thijssen MA, Swinkels DW, Ruers TJ, de Kok JB. Difference between free circulating plasma and serum DNA in patients with colorectal liver metastases. Anticancer Res 2002;22:421-5.

16. Pérez-Barrios C, Nieto-Alcolado I, Torrente M, Jiménez-Sánchez C, Calvo V, Gutierrez-Sanz L, et al. Comparison of methods for circulating cell-free DNA isolation using blood from cancer patients: impact on biomarker testing. Transl Lung Cancer Res 2016;5:665-72.

17. Camus V, Sarafan-Vasseur N, Bohers E, Dubois S, Mareschal S, Bertrand P, et al. Digital PCR for quantification of recurrent and potentially actionable somatic mutations in circulating free DNA from patients with diffuse large B-cell lymphoma. Leuk Lymphoma 2016;57:2171-9.
18. Li M, Xu C. Circulating cell-free DNA utility for the surveillance of patients with treated diffuse large B-cell lymphoma. Clin Oncol (R Coll Radiol) 2017;29:637-8.

19. Alvarado-Vásquez N. Circulating cell-free mitochondrial DNA as the probable inducer of early endothelial dysfunction in the prediabetic patient. Exp Gerontol 2015;69:70-8.

20. Naumann DN, Hazeldine J, Dinsdale RJ, Bishop JR, Midwinter MJ, Harrison P, et al Endotheliopathy is associated with higher levels of cell-free DNA following major trauma: A prospective observational study. PloS One 2017;12:e0189870.

21. Frank MO. Circulating cell-Free DNA differentiates severity of inflammation. Biol Res Nurs 2016;18:477-88.

22. Moreira VG, Prieto B, Rodríguez JS, Alvarez FV. Usefulness of cell-free plasma DNA, procalcitonin and C-reactive protein as markers of infection in febrile patients. Ann Clin Biochem 2010;47:253-8.

23. Pérez-Santiago J, Schrier RD, de Oliveira MF, Gianella S, Var SR, Day TR, et al. Cellfree mitochondrial DNA in CSF is associated with early viral rebound, inflammation, and severity of neurocognitive deficits in HIV infection. J Neurovirol 2016;22:191200.

24. Hohaus S, Giachelia M, Massini G, Mansueto G, Vannata B, Bozzoli V, et al. Cellfree circulating DNA in Hodgkin's and non-Hodgkin's lymphomas. Ann Oncol 2009;20:1408-13.

25. Manier S, Park J, Capelletti M, Bustoros M, Freeman SS, Ha G, et al. Whole-exome sequencing of cell-free DNA and circulating tumor cells in multiple myeloma. Nat Commun 2018;9:1691.

26. Oberle A, Brandt A, Voigtlaender M, Thiele B, Radloff J, Schulenkorf A, et al. Monitoring multiple myeloma by next-generation sequencing of $V(D)$ $\mathrm{J}$ rearrangements from circulating myeloma cells and cell-free myeloma DNA. Haematologica 2017;102:1105-11.

27. Gao YJ, He YJ, Yang ZL, Shao HY, Zuo Y, Bai Y, et al. Increased integrity of circulating cell-free DNA in plasma of patients with acute leukemia. Clin Chem Lab Med 2010;48:1651-6.

28. Duque-Afonso J, Waterhouse M, Pfeifer D, Follo M, Duyster J, Bertz H, et al. Cellfree DNA characteristics and chimerism analysis in patients after allogeneic cell transplantation. Clin Biochem 2018;52:137-41.

29. Aljurf M, Abalkhail H, Alseraihy A, Mohamed SY, Ayas M, Alsharif F, et al Chimerism analysis of cell-free DNA in patients treated with hematopoietic stem cell transplantation may predict early relapse in patients with hematologic malignancies. Biotechnol Res Int 2016;2016:8589270.

30. Herrera AF, Kim HT, Kong KA, Faham M, Sun H, Sohani AR, et al. Next-generation sequencing-based detection of circulating tumour DNA After allogeneic stem cell transplantation for lymphoma. Br J Haematol 2016;175:841-50. 